\title{
Modelling neutral beams in fusion devices: Beamlet-based model for fast particle simulations
}

\author{
O. Asunta ${ }^{\text {a,* }}$, J. Govenius ${ }^{\mathrm{a}}$, R. Budny ${ }^{\mathrm{b}}$, M. Gorelenkova ${ }^{\mathrm{b}}$, G. Tardini ${ }^{\mathrm{c}}$, T. Kurki-Suonio ${ }^{\mathrm{a}}$, A. Salmi ${ }^{\mathrm{d}}$, S. Sipiläa ${ }^{\mathrm{a}}$, the ASDEX \\ Upgrade Team $^{\mathrm{c}}$, and the JET EFDA Contributors ${ }^{1}$ \\ JET-EFDA, Culham Science Centre, OX14 3DB, Abingdon, UK
}

\begin{abstract}
${ }^{a}$ Department of Applied Physics, Aalto University, Association Euratom-Tekes, P.O. Box 14100, FI-00076 AALTO, Finland
${ }^{b}$ Princeton Plasma Physics Laboratory, P.O. Box 451, Princeton, New Jersey 08543-0451, USA

${ }^{c}$ Max-Planck-Institut für Plasmaphysik, IPP-EURATOM Association, D-85748 Garching bei München, Germany

${ }^{d}$ Association Euratom-Tekes, VTT, P.O. Box 1000, FIN-02044 VTT, Finland
\end{abstract}

\begin{abstract}
Neutral beam injection (NBI) will be one of the main sources of heating and non-inductive current drive in ITER. Due to high level of injected power the beam induced heat loads present a potential threat to the integrity of the first wall of the device, particularly in the presence of non-axisymmetric perturbations of the magnetic field. Neutral beam injection can also destabilize Alfvén eigenmodes and energetic particle modes, and act as a source of plasma rotation. Therefore, reliable and accurate simulation of NBI is important for making predictions for ITER, as well as for any other current or future fusion device. This paper introduces a new beamlet-based neutral beam ionization model called BBNBI. It takes into account the fine structure of the injector, follows the injected neutrals until ionization, and generates a source ensemble of ionized NBI test particles for slowing down calculations. BBNBI can be used as a stand-alone model but together with the particle following code ASCOT it forms a complete and sophisticated tool for simulating neutral beam injection. The test particle ensembles from BBNBI are found to agree well with those produced by PENCIL for JET, and those produced by NUBEAM both for JET and ASDEX Upgrade plasmas. The first comprehensive comparisons of beam slowing down profiles of interest from BBNBI+ASCOT with results from PENCIL and NUBEAM/TRANSP, for both JET and AUG, are presented. It is shown that, for an axisymmetric plasma, BBNBI+ASCOT and NUBEAM agree remarkably well. Together with earlier 3D studies, these results further validate using BBNBI+ASCOT also for studying phenomena that require particle following in a truly three-dimensional geometry.
\end{abstract}

Keywords: neutral beam injection, beamlet, Monte Carlo, fast ions

\section{Introduction}

Transport modelling of magnetically confined fusion plasmas relies on knowing the plasma profiles (density, temperature, momentum) and their sources: particles, heat and torque. The profiles themselves can be extracted from measurements, but the sources can not. Instead, they have to be simulated. Therefore, accurate modelling of sources is of paramount importance for transport modelling. Neutral beam injection is the main source of heating in most large tokamaks today and, furthermore, provides a significant and flexible source of torque and current. In particular, neutral beams' capability to drive off-axis current is of interest from the perspective of steadystate operating scenario development for tokamaks.

In the earlier stages of fusion research it was customary to assume that tokamak plasmas are toroidally symmetric. In practice, however, the finite number of toroidal field coils alone is enough to break the axisymmetry of the magnetic field. Even

\footnotetext{
*e-mail: otto.asunta@aalto.fi

${ }^{1}$ See the Appendix of F. Romanelli et al., Proceedings of the 24th IAEA Fusion Energy Conference 2012, San Diego, USA
}

the remaining toroidal periodicity is destroyed by toroidally localized magnetic perturbations caused by, e.g, other external coils. Also the first wall has a three-dimensional (3D) structure. Consequently, the tools used for modelling phenomena in such plasmas should be able to cope with a fully 3D system.

The particle following Monte Carlo code ASCOT [1, 2, 3] has been used to simulate NBI in several fusion devices including ITER [3], ASDEX Upgrade (AUG) [4], Joint European Torus (JET) [5, 6], DIII-D [7], and TEXTOR [8]. Until recently, however, the initial test particle ensemble for ASCOT had to be obtained from an external code, e.g. PENCIL [9] for JET and ITER, and FAFNER [10] for AUG. Compared to using these codes, having a detailed NBI model coupled to ASCOT has two major advantages. First, it guarantees that the magnetic and plasma backgrounds used for neutral beam ionization calculations are identical to those used for simulating the resulting fast particle ensemble. This is becoming increasingly important with the use of truly three-dimensional magnetic fields and the prospect of using two-dimensional neutral and plasma densities and temperatures. Second, having a purpose-built model offers greater flexibility; the same model can be used for a number of existing devices and even for developing and studying NBI 
geometries for devices that do not yet exist.

Based on the considerations above, a beamlet-based NBImodel called BBNBI has been developed. It uses the same I/O structures as ASCOT and can therefore handle complex magnetic geometries and ionization outside the last closed flux surface (provided that plasma temperature and density there are known). While BBNBI was designed to satisfy the needs of ASCOT, it is a completely independent tool. Thanks to this modularity, BBNBI will be utilized in the EFDA Integrated Tokamak Modelling framework (ITM) [11] as a stand-alone actor for calculating the neutral beam ionization. Within the framework, BBNBI can then be combined with any fast ion slowing down code compatible with the ITM structures.

Section 2 introduces the beamlet-based NBI model. In Sec. 3.1 the neutral beam ionization predicted by BBNBI is compared against results of established NBI codes NUBEAM [12, 13], the NBI module of transport code TRANSP [14], and PENCIL [9]. FAFNER was omitted from this comparison because it is no longer supported at AUG and its results have been shown to be in good agreement with those of NUBEAM [15]. Section 3.2 goes beyond beam ionization by studying the steady-state slowing down profiles of beam ions. The fast ion ensembles produced by BBNBI are followed with ASCOT and the resulting slowing down profiles extensively compared to those from NUBEAM and PENCIL. The purpose of this benchmark is (i) to quantify the effect that minor differences in the initial test particle ensemble have on the actual quantities of interest, and (ii) to validate the use of BBNBI together with ASCOT for NBI modelling. The results of this work are summarized in Sec. 4.

\section{Description of BBNBI}

BBNBI follows neutrals from the injector until they are ionized, hence producing an ensemble of fast test ions. BBNBI is an independent tool, but it was designed to satisfy the needs of the particle following Monte Carlo code ASCOT and even uses the same magnetic and plasma input. Because ASCOT is often used for simulating fast particle wall losses, the magnetic backgrounds used need to extend all the way to the walls of the device. Consequently, unlike codes that have earlier been used for beam ionization, BBNBI can take into account ionization even outside the last closed flux surface.

\subsection{Describing the injector geometry}

Neutral beam injectors in all large tokamaks are based on a similar beamline structure: an ion source connected to an electrostatic accelerator is followed by a neutralizer and a residual ion dump [16]. BBNBI follows the neutral particles starting from the grounded grid, i.e., the last accelerator grid. The beam is modelled as a set of sub-beams, or beamlets, one from each grid hole in the grounded grid, as shown in Fig. 1(a). The fine structure of the beam is readily taken into account by defining the location and direction of each beamlet individually. Normally the direction of each beamlet is calculated from the orientation and the vertical (see Fig. 1(b)) and horizontal focal

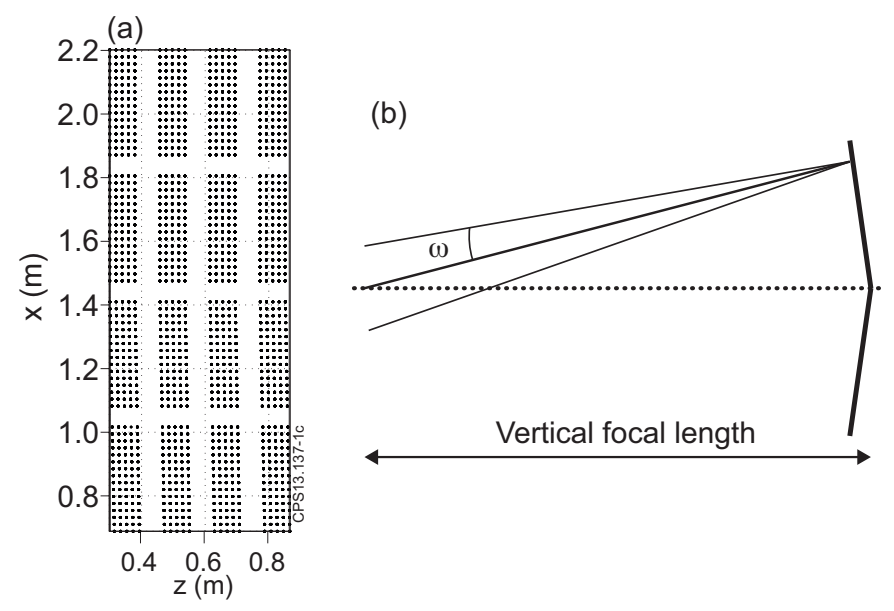

Figure 1: (a) a front view of ITER grounded grid as seen by BBNBI and (b) a side-view of the grounded grid of a JET PINI (Positive Ion Neutral Injector) with tilted grid halves showing the direction of a single beamlet and a sampla deviation angle $\omega$. The vertical focal length, i.e., the distance where the beamlets are focused in the the vertical direction, is also shown.

lengths of the beam. Defining the geometry on a beamlet-bybeamlet basis allows including arbitrary device specific features to the model, such as the tilt between the upper and lower halves of the injector grids of JET and AUG. The other adjustable parameters for each injector unit are:

- Injected particle species $(\mathrm{H} / \mathrm{D} / \mathrm{T})$

- Total power

- Full energy of the beam particles, $E_{\max }$

- Fractions of particles in the different energy components $E_{\max }, E_{\max } / 2$, and $E_{\max } / 3$

In addition, beamlet divergence and the probability with which a neutral is injected from a given beamlet can be specified. For the simulations in this work, these probabilities were uniform. The aperture through which the beam must pass and/or material obstacles along the beamline can also be defined.

Beamlet divergence describes the power density $P(\omega)$, and hence also the particle density, as a function of the deviation angle $\omega$ from the beamlet direction (see Fig. 1(b)). Typically it is defined simply as a $1 / e$ width of a symmetric Gaussian or bi-Gaussian distribution around the beamlet axis. For example, for ITER the divergence is assumed to be a bi-gaussian that consists of a core and a halo part [17]:

$$
P(\omega) / P_{t o t}=\left[\frac{1-f}{\pi\left(X \omega_{\mathrm{c}}\right)^{2}} e^{-\left(\omega / \omega_{\mathrm{c}}\right)^{2}}+\frac{f}{\pi\left(X \omega_{\mathrm{h}}\right)^{2}} e^{-\left(\omega / \omega_{\mathrm{h}}\right)^{2}}\right],
$$

where $f$ is the fraction of power carried by the halo $(15 \%), \omega_{\mathrm{c}}$ and $\omega_{\mathrm{h}}$ are the $1 / e$ widths of the core and the halo (in the standard case $5 \mathrm{mrad}$ and $15 \mathrm{mrad}$, respectively), and $X$ is the distance from the grounded grid. When defining the divergence, the absolute values of the distribution and, therefore, the variable $X$ in Eq. (1), are insignificant because the distribution is 
only used to define the relative probabilities of different deviation angles $\omega$. BBNBI assigns each test particle a unique direction so that the ensemble as a whole follows the given diverging power distribution.

\subsection{Generating the test particle ensemble}

To generate an NBI test particle, a neutral particle from a random beamlet is chosen. The neutral is assigned a velocity in the direction of the beamlet, offset according to the beamlet divergence, and moved along its velocity vector until it either hits an obstacle or enters the vacuum chamber. Typical obstacles include beam scrapers and the edges of an aperture through which the beam must pass.

Inside the device the neutral particle is assigned a uniformly distributed random ionization threshold $\lambda \in[0,1]$ and it is advanced along a straight trajectory while simultaneously evaluating the cumulative ionization probability $P$. Reaction rates $R_{\mathrm{r}}$ of the neutral atom with the plasma determine $P$ according to

$$
\begin{array}{r}
\frac{\mathrm{d}(1-P)}{\mathrm{d} s}=-\sum_{\mathrm{r}}(1-P) R_{\mathrm{r}}(s) \\
\Rightarrow P(s)=1-\exp \left(-\int_{0}^{s} \Sigma\left(s^{\prime}\right) \mathrm{d} s^{\prime}\right),
\end{array}
$$

where $s$ is the distance along the trajectory and $\Sigma\left(s^{\prime}\right)=$ $\sum_{\mathrm{r}} R_{\mathrm{r}}\left(s^{\prime}\right)$ is the total effective ionization cross-section. The distance dependence of the reaction rates originates from the position dependence of plasma parameters (densities, temperatures, impurities).

In the code the integral is discretized into small steps such that $\Sigma(s)$ can be taken constant between adjacent points $s_{\mathrm{i}}$ and $s_{i+1}$. In this limit $\Sigma(s)$ can be computed from the fits given by Suzuki et al. [18]. The probability $P_{\mathrm{i}}$ of ionization before $s_{\mathrm{i}}$ is then determined by

$$
1-P_{\mathrm{i}}=\left(1-P_{\mathrm{i}-1}\right) e^{-\left(s_{\mathrm{i}}-s_{\mathrm{i}-1}\right) \Sigma_{\mathrm{i}}},
$$

where $P_{0}=0$ and $\Sigma_{\mathrm{i}}=\left(\Sigma\left(s_{\mathrm{i}-1}\right)+\Sigma\left(s_{\mathrm{i}}\right)\right) / 2$. Once $\left(1-P_{\mathrm{i}}\right)$ falls below the random threshold $\lambda$, the last step is retaken and the exact ionization point $s_{f}$ is computed from

$$
s_{f}-s_{\mathrm{i}-1}=-\frac{1}{\Sigma_{\mathrm{i}}} \ln \left(\frac{\lambda}{1-P_{\mathrm{i}-1}}\right) \text {. }
$$

After this step a test particle is recorded. If the wall is encountered before $1-P_{\mathrm{i}}<\lambda$, the neutral particle is considered shinethrough.

The NBI geometries of AUG, DIII-D, FAST, ITER, JET, TEXTOR, MAST, and Tore Supra have already been implemented inside BBNBI. There should be no major obstacles in adding more devices. When used within the ITM framework [11], BBNBI ignores the internal implementation of the NBI geometry and, instead, requires the geometry as part of the input.

\section{Comparisons against established NBI codes}

NUBEAM, the NBI module of TRANSP suite of codes [14, 19], is one of the most widely used tools for NBI modelling. It has been extensively used for modelling both AUG [20, 21] and JET [22, 23, 24] plasmas. PENCIL [9] on the other hand, has been the standard tool for simulating neutral beams at JET since early 1990s. In this section, results of these established codes are compared to those of BBNBI for neutral beam ionization and ASCOT for the slowing down of the NBI ions.

The method for generating the initial test particle ensemble inside NUBEAM is similar to that of BBNBI, described in Sec. 2. There are, however, some minor differences in the injection geometry. For example, NUBEAM positions the beamlets randomly on a flat source grid, whereas BBNBI specifies the exact locations of the beamlets (i.e., the holes in the grounded grid) and as a result allows, e.g., the grid halves to be tilted (recall Fig. 1).

The ionization cross-sections are also calculated differently in BBNBI and NUBEAM. BBNBI uses parametrized crosssections by Suzuki et al. [18], whereas NUBEAM can use cross-sections from either ADAS [25], or Janev et al. [26]. It was, however, discovered that all three models produce very similar cross-sections, and NUBEAM ionization results using the two models are practically indistinguishable. This might not be the case for, e.g., higher plasma densities though, as shown by Kraus [15]. Still, for the purposes of this work, the two models were identical and the default cross-sections from ADAS were used in all the presented NUBEAM simulations.

Both ASCOT and NUBEAM are test particle following Monte Carlo (MC) codes. They integrate the equation of motion of the particle's guiding center (GC) in time and model the Coulomb collisions with the background plasma using MC collision operators for slowing down and pitch angle scattering [27]. ASCOT is also capable of following the particle's full orbit (FO), but due to its high computational cost and minimal effect on the results of the comparisons presented here, full orbit following was not used in this work. While NUBEAM follows the particle GC, it takes particles' finite Larmor radius into account by calculating fast ions' interactions with the plasma at a random position on the Larmor orbit instead of the GC location [13]. For ASCOT the interactions are calculated at the GC position.

PENCIL is a Fokker-Planck code that uses a simplified vessel structure, parametrized plasma equilibrium and a set of parallel pencils to model the beams. Due to the above mentioned simplifications, PENCIL is computationally very efficient and, nevertheless, often gives an adequate picture of the neutral beams. Earlier, PENCIL has also been used for providing an initial NBI test particle ensemble for neutral beam slowing down simulations performed with ASCOT.

The neutral beam injection geometries of both AUG and JET (cf. Fig. 1 in Refs. [28] and [29], respectively) have been modelled in detail in BBNBI as described in Sec. 2.1. In order to exclude discrepancies arising from differing plasma parameters, the ion and electron temperatures and densities were kept constant in time in the NUBEAM/TRANSP simulations and ex- 
ported from TRANSP output to BBNBI, ASCOT and PENCIL. The axisymmetric equilibrium and a broken line representation of the first wall of the device were extracted from TRANSP. Plasma rotation was set to zero because its effects on ionization and slowing down of the beam particles is not yet taken into account by BBNBI+ASCOT. Also charge-exchange (CX) reactions between the fast ions and the thermal neutrals were disregarded.

\subsection{Beam ionization}

In this section, it is shown that the results of the beam injection and ionization code BBNBI are in good quantitative agreement with NUBEAM for AUG neutral beams, and with NUBEAM and PENCIL for JET neutral beams. Because of the presence of $\mathrm{D}^{+}, \mathrm{D}_{2}^{+}$, and $\mathrm{D}_{3}^{+}$ions in the positive ion source, Positive Ion Neutral Injectors (PINIs) inherently produce three beam components with energies $E_{\max }, E_{\max } / 2$, and $E_{\max } / 3$. However, in the first benchmark, AUG beams were assumed monoenergetic with $E=E_{\max }$. This was done to avoid the risk of the energy component mix masking possible discrepancies caused by, e.g., the beam geometry or the different ionization cross-section models in the two codes. For JET, beams with all three energy components were used.

For the first comparison, 200000 test particles with $E=$ $60 \mathrm{keV}$ were injected from AUG PINIs 1-4, and an equal number of $93 \mathrm{keV}$ test particles from PINIs 5-8, using both BBNBI and NUBEAM. The total power injected from each PINI was $2.5 \mathrm{MW}$ and the plasma parameters were: $I_{p}=0.8 \mathrm{MA}$, $B_{T}=2.7 \mathrm{~T}$, and $n_{e}(0)=7.1 \times 10^{19} \mathrm{~m}^{-3}$.

The present day tools for studying transport processes in tokamak plasmas tend to operate in only one dimension. Therefore, the densities of ionized particles from all the PINIs are presented in Fig. 2 as a function of the radial coordinate $\rho_{\text {pol }}=$ $\sqrt{\left(\Psi-\Psi_{\text {axis }}\right) /\left(\Psi_{\text {sep }}-\Psi_{\text {axis }}\right)}$, i.e. the square root of the normalized poloidal magnetic flux. For all the PINIs, the density profiles of the ionized beam particles agree remarkably well.

Due to different ionization cross-sections used in the two codes, NUBEAM predictions for beam shine-through are about $10 \%$ larger than those of BBNBI. However, the effect of this discrepancy is only visible for the most 'radial' (i.e. pointing toward to central column of the device) high energy PINIs 5 and 8 with the highest shine-through fractions of 6-7\% of injected power (see Fig. 2(e) and (h)). For the rest of the PINIs, the shine-through is less than $4 \%$ of the injected power.

Even though the radial profiles are of most interest from the transport analysis point of view, there are other applications where the actual three-dimensional shape of the beam might be of importance. For example, when calculating the fast particle wall loads in the presence of magnetic perturbations, the three-dimensional nature of the beams plays a crucial role. For AUG, the beam shapes from the two codes are nearly identical for all the eight PINIs in both the poloidal and the toroidal cross-sections of the device. This is demonstrated for two representative PINIs 4 (60 keV, 'radial') and 6 (93 keV, 'current drive'), in Figures. 3 and 4.

Initial velocity of the ionized particles is another key factor in predicting the effects of NBI. The speed of a particle is
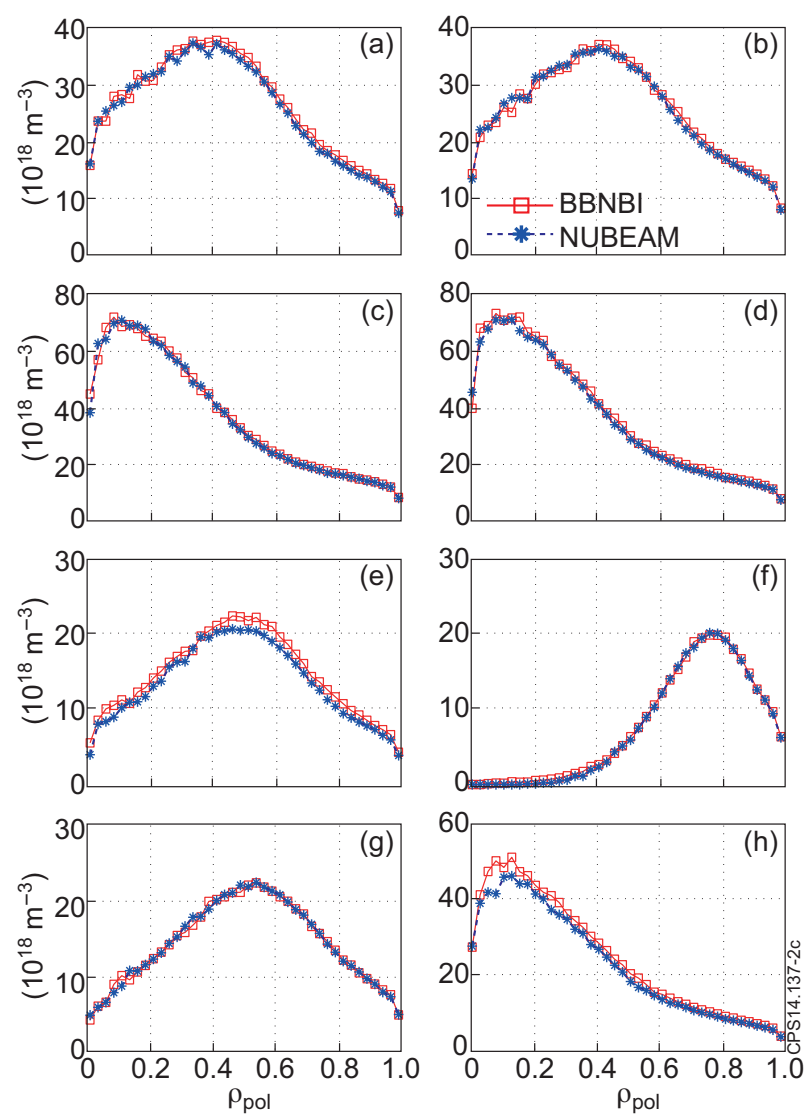

Figure 2: Densities of ionized beam neutrals as a function of the radial coordinate $\rho_{\text {pol }}$ for AUG PINIs $1-8$, corresponding to panels (a)-(h). Following the naming conventions of Fig. 1 in Ref. [28], PINIs 1 and 4 are 'radial' (small tangency radii, pointing toward the central column), PINIs 2, 3, 5, and 8 are 'tangential' (larger tangency radii, partly hitting the central column), and PINIs 6 and 7 are called 'current drive' PINIs (even larger tangency radii). The vertical aiming of the beams, however, results in similar radial density profiles of ionized particles for characteristically different beams, such as PINIs 5 and 7.

fixed by its kinetic energy. Therefore, particle pitch $\xi=v_{\|} / v$, where $v_{\|}$is the particle velocity parallel to the magnetic field, is enough to define the parallel and perpendicular velocity components. Figure 5 shows a good agreement between BBNBI and NUBEAM in the initial particle distribution in $\left(\rho_{\mathrm{pol}}, \xi\right)$. Together with the earlier figures of particle densities it confirms the excellent overall match between the AUG NBI particle ensembles produced by the two codes.

There are two known differences that cause the minor discrepancies between the particle distributions from BBNBI and NUBEAM: (i) the ionization cross-sections used in the codes (discussed above in Sec 3), and (ii) the modelling of beam scraping.

Both BBNBI and NUBEAM have the option to take into account the finite size of the beam port and other elements limiting the beam shape by scraping it along the way. In BBNBI these elements are automatically defined in fixed coordinates with respect to the device and, consequently, all the PINIs of a given injector see the same scrapers. NUBEAM, on the other hand, defines individual beam scrapers separately for each PINI. In NUBEAM the scrapers are, by default, centered around the 

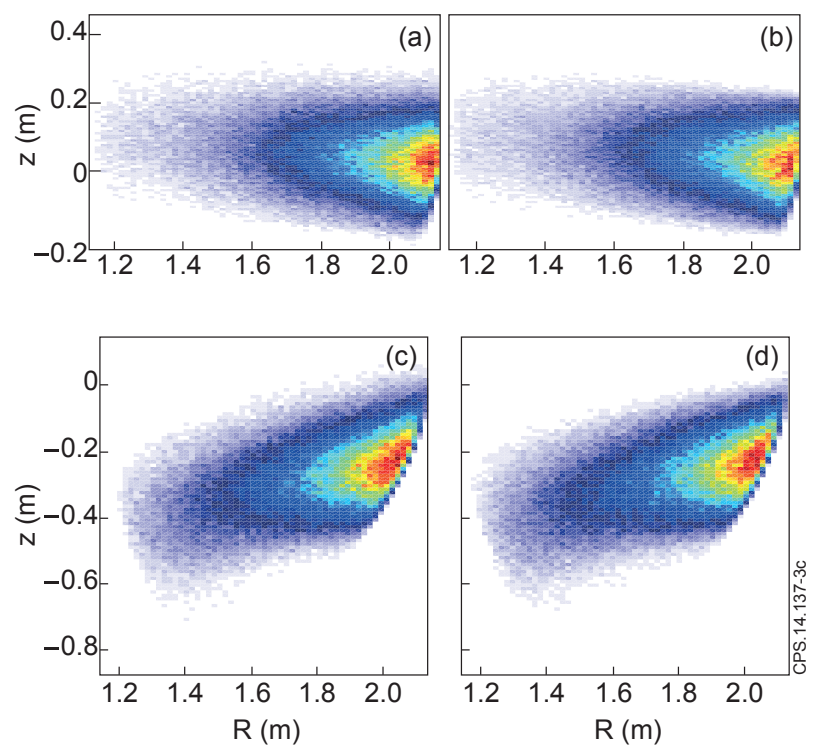

Figure 3: Two-dimensional histograms of the ionization locations of NBI particles injected from the AUG PINIs 4 (top panels) and 6 (bottom panels) projected to the poloidal $(R, z)$ plane: BBNBI (left column), and NUBEAM (right). The plots within each row are plotted using the same colormap.

$\left(\times 10^{17}\right)$



Figure 4: Two-dimensional $(x, y)$ histograms of the ionization locations of NBI particles injected from the AUG PINIs 4 and 6: NUBEAM results are presented as white contours overlaid on top of the BBNBI results presented with the surface color.

beamline. As a result, depending on the aiming of the beams, they can be scraped in different ways by the two codes. For the results presented here, the scrapers in BBNBI were set so large that all the beams pass through them nearly unscraped, as they do in the corresponding NUBEAM simulations.

For JET neutral beams, a comparison similar to the AUG benchmark discussed above was performed. For JET however, a more realistic setup was adopted by taking into account all

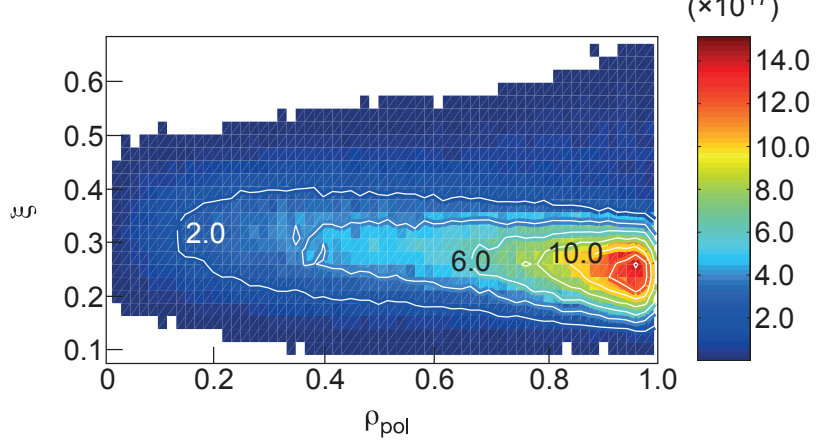

$\left(\times 10^{17}\right)$

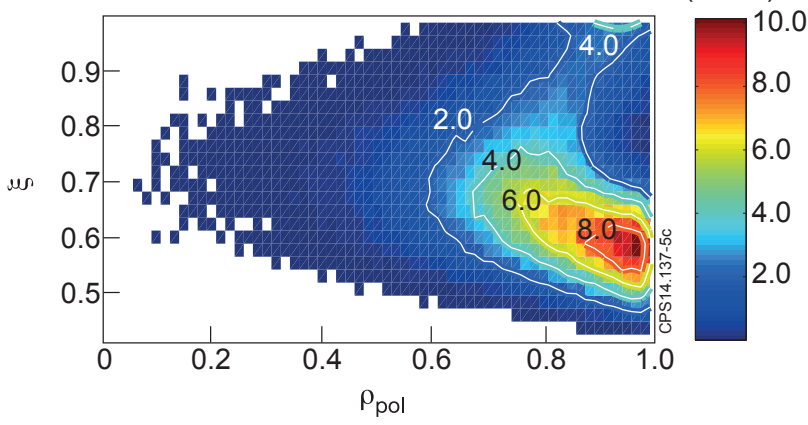

Figure 5: Two-dimensional $(\rho, \xi)$ histograms of the ionization locations of NBI particles injected from the AUG PINIs 4 (top) and 6 (bottom): NUBEAM results are presented as white contours overlaid on top of the BBNBI results presented with the surface color.

three energy components that the positive ion neutral injectors inherently produce. The beam power fractions used in this work for $E_{\max }: E_{\max } / 2: E_{\max } / 3$ were $84 \%: 12 \%: 4 \%$. The beams were injected to a JET-like plasma with $I_{p}=2.1 \mathrm{MA}, B_{T}=2.3 \mathrm{~T}$, and $n_{e}(0)=2.3 \times 10^{19} \mathrm{~m}^{-3}$

In order to compare the beam ionization in JET, 200000 test particles with maximum energy $E_{\max }=100 \mathrm{keV}$ and the total power of 1.0 MW were injected from all eight PINIs in the octant 8 injector using BBNBI and NUBEAM. For plotting purposes, the same number of test particles were also generated for each PINI from the PENCIL output. PINIs 1, 2, 7, and 8 are 'tangential' (larger tangency radii), whereas PINIs 3, 4, 5, and 6 are 'normal' (smaller tangency radii, pointing toward the central column) using the nomenclature of Fig. 1 of Ref. [29].

The densities of ionized particles as a function of $\rho_{\text {pol }}$ for each PINI are plotted Fig. 6. The agreement between BBNBI, NUBEAM, and PENCIL is excellent. PENCIL beams are marginally narrower and, consequently, more peaked in $\rho_{\text {pol }}$, but the difference is tiny.

The main characteristic difference between BBNBI and PENCIL is manifested in Fig. 7 which portrays the 2D histograms of the particles' ionization location in $(R, z)$ plane. The PENCIL beams clearly have an internal structure due to the small number of pencils used, whereas the beams created by BBNBI and NUBEAM are smoother and have no such artificial structures. However, these artifacts are not critical because nowadays PENCIL is exclusively used for 1D beam slowing down simulations where the artifacts are washed out (see 

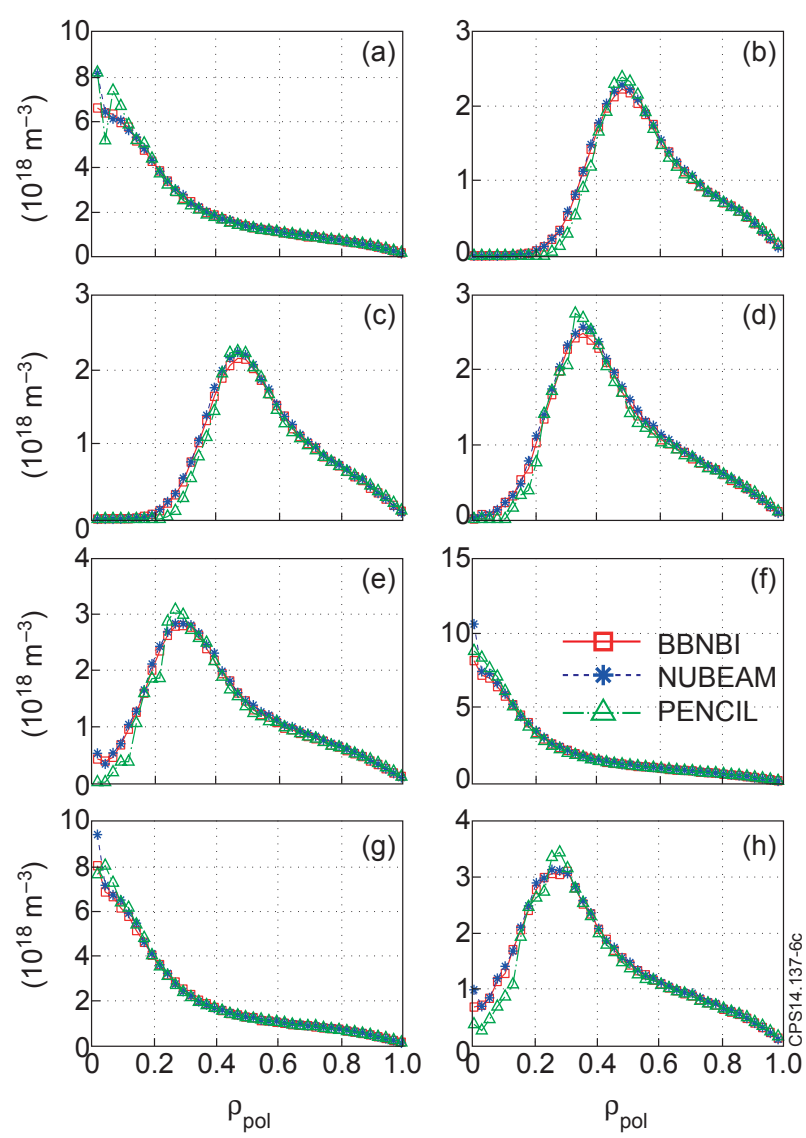

Figure 6: Densities of ionized beam neutrals from JET octant 8 PINIs 1-8, corresponding to panels (a)-(h), injected by BBNBI, NUBEAM, and PENCIL.
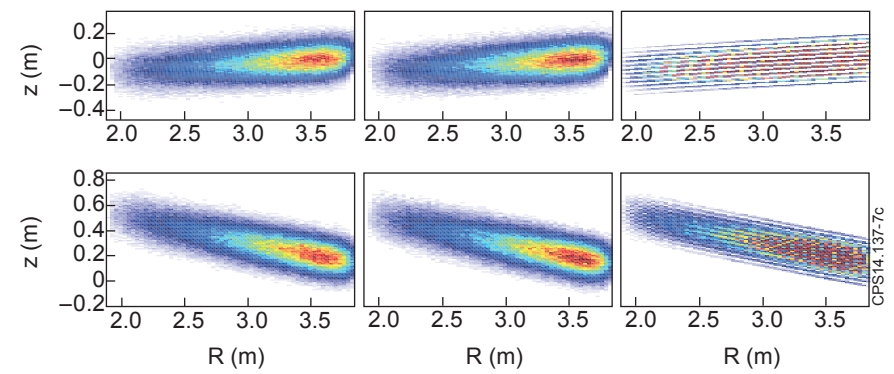

Figure 7: Two-dimensional histograms $(R, z)$ of the ionization locations of NBI particles from BBNBI (left column), NUBEAM (middle), and PENCIL (right) for PINIs 4 (top row) and 6 (bottom row) in a JET-like plasma. The plots within each row are plotted using the same colormap.

Fig. 6).

\subsection{NBI ion slowing down simulations}

Slowing down simulations of ionized NBI particles were performed for both JET and AUG using the test particle ensembles obtained in Sec. 3.1. The results of ASCOT and NUBEAM were found to agree very well for both devices, whereas PENCIL results for JET differ slightly from the two.

For one-dimensional transport simulations, good estimates for the particle, heat, current, and momentum sources due to NBI are needed. Because the sources can affect the plasma
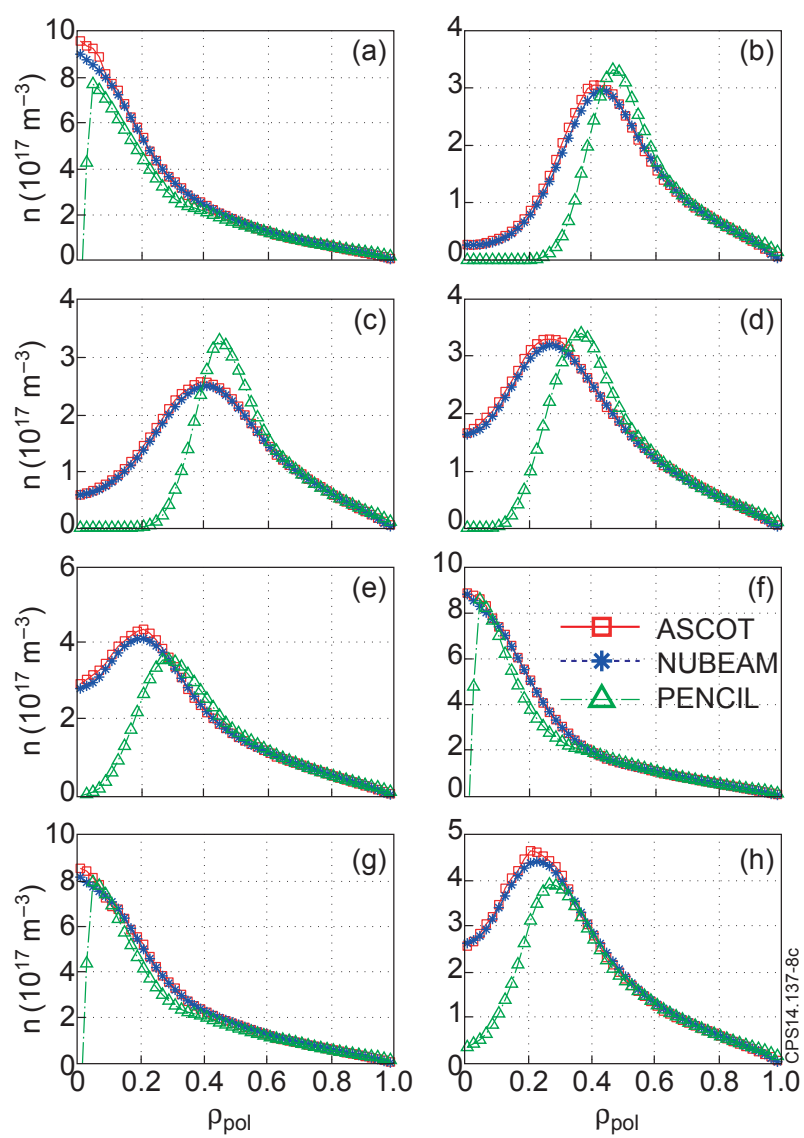

Figure 8: Fast ion slowing down density in a JET-like plasma for octant 8 PINIs $1-8$, corresponding to panels (a)-(h).

temperature and density and, consequently, the ionization and slowing down of the neutral beam, the NBI source has to be operated in a continuous fashion, with thermalized particles leaving and freshly ionized ones continuously entering the system. This is how NUBEAM operates within TRANSP, and also how ASCOT operates when it is used as the fast ion module within the JINTRAC simulation environment $[30,31]$ and, in the future, within the European Transport Simulator (ETS) [32, 33].

In this work, however, we want to find the steady-state profiles of various quantities of interest. They are obtained by running the codes until the full slowing-down distribution has built up, i.e., for several slowing-down times. In practice, to get statistically robust results, NUBEAM profiles were averaged over three seconds of simulation after the build-up of the full slowing-down distribution.

ASCOT also offers an alternative, faster route to the fast ion steady-state profiles. Instead of a continuous source, all the injected particles are launched in the beginning of the simulation and their contribution to the quantities of interest are accumulated until they have thermalized. Throughout this work, in all the three codes, the particles were deemed thermalized and their simulation ended when their energy dropped below 1.5 times the local ion temperature.

The first slowing down comparison was performed for a JETlike plasma with $I_{p}=2.1 \mathrm{MA}, B_{T}=2.3 \mathrm{~T}$, and $n_{e}(0)=$ 

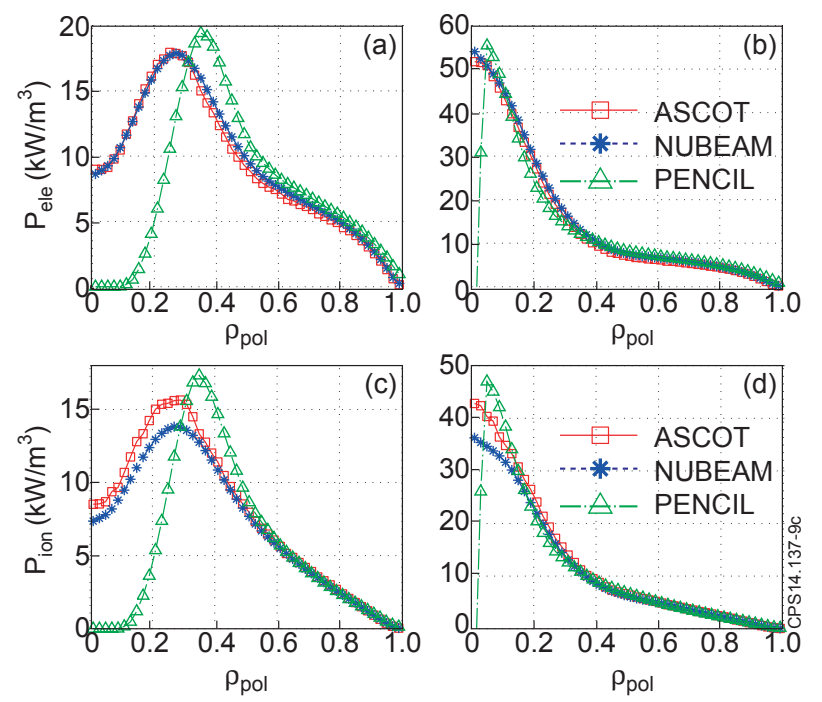

Figure 9: Power deposition from the beam particles to the thermal electrons (top row) and ions (bottom row) in a JET-like plasma for octant 8 PINIs 4 (left column) and 6 (right column).

$2.3 \times 10^{19} \mathrm{~m}^{-3}$. The fast particle densities from the three codes for the eight octant 8 PINIs are plotted in Fig. 8. The agreement between ASCOT and NUBEAM is very good. PENCIL ignores the ion orbit effects and therefore the peak of the fast ion density profile in Fig. 8 has not moved in $\rho_{\text {pol }}$ compared to the initial particle distribution plotted in Fig. 6. Consequently, its agreement with ASCOT and NUBEAM is rather poor, particularly for the 'normal' off-axis PINIs 3 and 4 that inject a larger fraction of the particles to banana orbits. It should be noted, however, that the densities predicted by the three codes are very similar at the outer parts of the plasma $\left(\rho_{\text {pol }}>0.4\right)$ for all but the most off-axis PINIs 2, 3 and 4 . In addition, the very core is insignificant in terms of total particle numbers due to the increasing volume differentials towards the last closed flux surface.

While all the PINIs were examined, from now on the plots that are shown will be limited to only two representative PINIs: PINI 4 (off-axis, normal) and PINI 6 (on-axis, normal). This is done because the results of PINIs 2, 3, 5, and 8 behave similarly to those of PINI 4, whereas the results of PINIs 1 and 7 behave similarly to PINI 6. Therefore, the conclusions drawn for PINIs 4 and 6 can be straightforwardly extended to cover the rest of the JET PINIs.

Traditionally the primary purpose of neutral beams in fusion devices has been heating the plasma. As the injected particles traverse the plasma, their energy is transferred to the thermal electrons and ions through Coulomb collisions. Figure 9 shows the power deposition from the steady-state distribution of NBI ions to electrons ((a) and (b)) and ions ((c) and (d)). Again, ASCOT and NUBEAM produce similar results, whereas PENCIL profiles are more peaked. Unsurprisingly, the shapes of the power deposition profiles follow closely the shapes of the density profiles shown in Fig. 8.

The total deposited powers to thermal electrons and ions are presented in Table 1. PENCIL consistently deposits more
Table 1: Total power depositions from NBI ions from JET octant 8 PINIs 4 and 6 to the thermal electrons and ions predicted by ASCOT, NUBEAM and PENCIL.

PINI 4 PINI 6

\begin{tabular}{lll} 
Power to electrons (MW) & & \\
\hline ASCOT & 0.43 & 0.44 \\
NUBEAM & 0.44 & 0.45 \\
PENCIL & 0.50 & 0.50
\end{tabular}

Power to ions (MW)

\begin{tabular}{lll}
\hline ASCOT & 0.31 & 0.32 \\
NUBEAM & 0.29 & 0.30 \\
PENCIL & 0.31 & 0.33
\end{tabular}
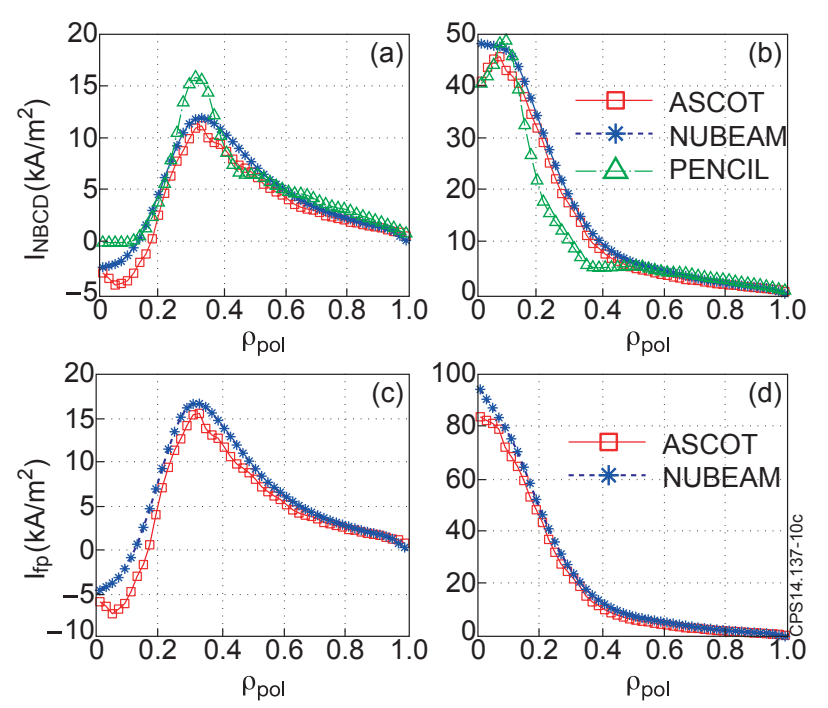

Figure 10: Neutral beam driven current after taking the electron shielding into account, (top row) and unshielded fast ion current (bottom row) in a JET-like plasma for octant 8 PINIs 4 (left column) and 6 (right column).

power to the plasma because it predicts less shine-through and fast particle losses. The minor discrepancy between ASCOT and NUBEAM in how the deposited power is divided to thermal ions and electrons, on the other hand, is due to the different implementations of the MC collision operators. NUBEAM does not separate the power deposited to the fuel and impurity ions, but the difference in ASCOT and NUBEAM power deposition profiles to ions (see Figs. 9(c) and (d)) is equal to the ASCOT prediction of the power deposited to the plasma impurities.

The ability of neutral beams to drive (off-axis) current is of great interest because of its importance for scenario development and steady-state scenarios in particular. All three codes compared in this work routinely provide a radial profile of the current driven by the NBI ions. The driven current is calculated from the fast ion current by multiplying it by an electron shielding factor. To calculate this factor ASCOT used the model by Mikkelsen and Singer [34], whereas for the NUBEAM simulations presented here the model by Lin-Liu and Hinton [35] was used. The model PENCIL uses for calculating the driven cur- 

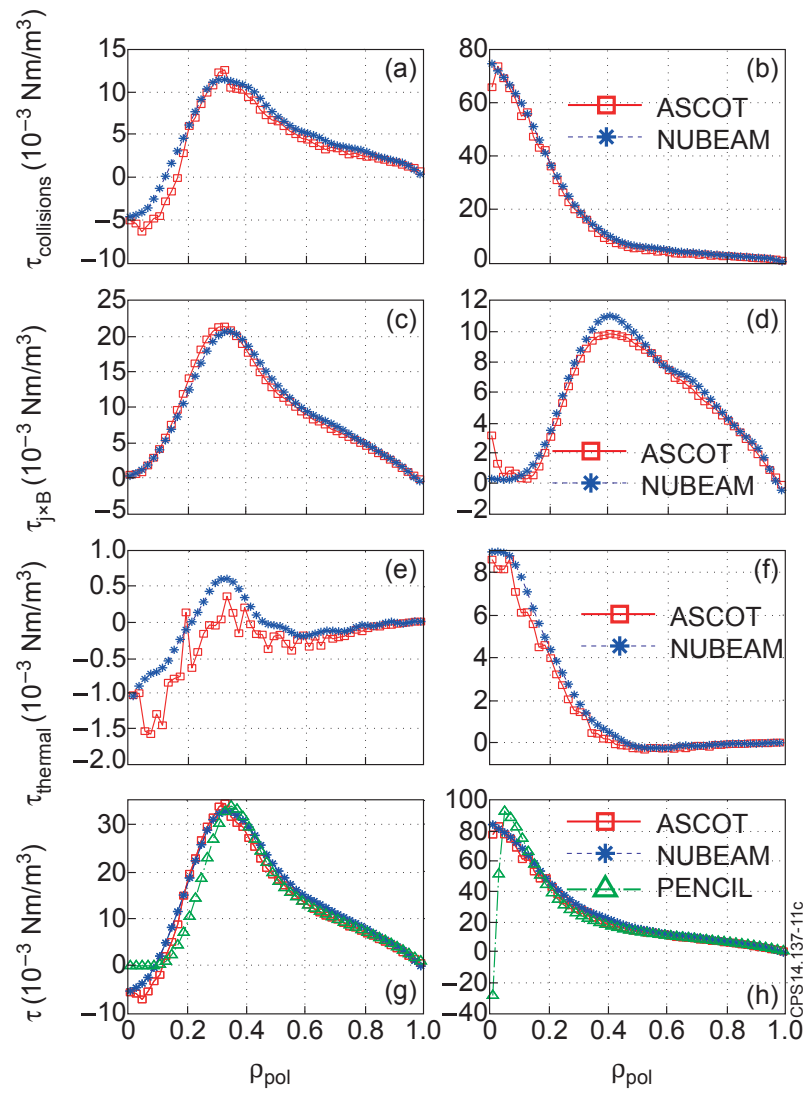

Figure 11: Collisional torque induced by beam ions (top row), $\vec{j} \times \vec{B}$ torque induced by beam ions (second row), the residual torque carried by the thermalized beam ions (third row), and total torque induced by beam ions (bottom row) in a JET-like plasma for octant 8 PINIs 4 (left column) and 6 (right column).

rent from the fast ion current is described in Refs. [9] and [36].

The neutral beam driven currents given by ASCOT, NUBEAM and PENCIL are plotted in the top row of Fig. 10. The profiles match well, even though PENCIL results are again more peaked than those of the other two codes. In addition to the driven current, ASCOT and NUBEAM also output the fast ion current, i.e. the quantity collected during simulations. Comparing the fast ion current profiles (bottom row of Fig. 10) with the profiles of the driven current (top row of Fig. 10) reveals that the current drive models used by ASCOT and NUBEAM give similar results, at least for the plasma conditions used in this work.

The small negative currents close to the magnetic axis for PINI 4 in Fig. 10(a) and (c) are due to the return legs of banana orbits. Hence, they only occur for the normal PINIs that have their peak density far enough from the magnetic axis (i.e. PINIs 3 and 4). During this work it was discovered that the magnitude of this negative current density depends strongly on the beam width in $\rho_{\text {pol }}$ : making the beam marginally narrower produced larger negative current densities. This makes accurate predictions of the beam ionization critical. While the total negative current is small and of little importance in JET, this effect could potentially be utilized for $q$-profile tailoring in future fusion devices.

In recent years, the transport of toroidal momentum in toka- mak plasmas has been an active field of research [22, 37] because of its importance for plasma stability. Therefore, the sources for toroidal momentum have to be understood. The torque applied by the neutral beams on the plasma can be divided in three main components [38]: (i) collisional torque due to the transfer of toroidal momentum from the NBI ions to the thermal bulk in collisions, (ii) $\vec{j} \times \vec{B}$ torque due to radial excursions of the NBI ions, and (iii) thermalization torque due to the toroidal momentum carried by the NBI ions when they have been thermalized. These three components calculated by ASCOT and NUBEAM are depicted individually in top three rows of Fig. 11. Their sums are shown in the bottom row of the same figure together with the total torque given by PENCIL.

The collisional torques calculated by ASCOT and NUBEAM (see Figs. 11 (a) and (b)) are very similar. Also the $\vec{j} \times \vec{B}$ torques, plotted in Fig. 11(c) and (d) nearly overlap. Positive $\vec{j} \times \vec{B}$ torque is caused by an ion moving radially inwards and is to be expected because the NBI ions injected in the direction of the plasma current (co-current injection) are born on the outer leg of banana orbits. That is, the particle will on average move inwards on its first orbit after ionization.

The collisional and $\vec{j} \times \vec{B}$ torques are both calculated as a sum over a large number of time steps. Thermalization torque, on the other hand, is calculated only once for each test particle. As a result, the ASCOT profiles plotted in Fig. 11(e) and (f) are rather noisy, whereas the time averaging performed for NUBEAM profiles helps making it relatively smooth. The general trends are nevertheless visible and similar for the two codes, even if they don't quite coincide for off-axis PINIs, like the plotted PINI 4. The discrepancy is, however, of little practical importance as the thermalization torque accounts for only a few percent of the total torque. Even locally it rarely exceeds $10 \%$ of the combined effect of the collisional and the $\vec{j} \times \vec{B}$ torques.

The total torques calculated by ASCOT and NUBEAM, presented at the bottom row of Fig. 11, are very similar despite the minor differences in the $\vec{j} \times \vec{B}$ torques and the noise and uncertainties in the thermalization torques. Furthermore, even PENCIL produces torque profiles that are in reasonable agreement with ASCOT and NUBEAM regardless of its simplifications. PENCIL assumes all the initial toroidal momentum of the beam ions to be deposited on the flux surface where the injected neutrals are ionized. For the particle following codes, the combination of finite orbit widths and collisional transport of the fast ions results in a very similar total torque profile to the one predicted by PENCIL.

The same slowing down comparisons that were presented above for PINIs in JET octant 8 injector were also performed for the eight AUG PINIs for a plasma with $I_{p}=0.8 \mathrm{MA}$, $B_{T}=2.7 \mathrm{~T}$, and $n_{e}(0)=7.1 \times 10^{19} \mathrm{~m}^{-3}$. The AUG NBI geometry is presented in Fig. 1 of Ref. [28]. The fast ion densities for all AUG PINIs, plotted in Fig. 12, show a very good correspondence between ASCOT and NUBEAM. Marginally higher particle losses result in NUBEAM predicting slightly lower densities for PINIs 6 and 7 than ASCOT (see Fig. 12(f) and (g)). Comparing the fast ion densities in Fig. 12 to the initial densities of ionized particles presented in Fig. 2, it is clear that minor 



Figure 12: Fast ion slowing down density in a AUG-like plasma for PINIs 1-8, corresponding to panels (a)-(h).

differences in the shine-through for PINIs 5 and 8 do not have a significant impact on the resulting fast ion source; ASCOT and NUBEAM results for those PINIs overlap, except for the core plasma. It should be reiterated that in terms of total particle numbers the very core is insignificant due to the increasing volume differentials towards the last closed flux surface.

For power deposition to the plasma electrons and ions, beam driven current, and total torque induced by the beam ions, only the results for two representative PINIs: PINI 4 (60 keV, 'radial') and PINI 6 (93 keV, 'current drive') are shown in Fig. 13. However, the good agreement between ASCOT and NUBEAM shown for PINIs 4 and 6 extends to all AUG PINIs. The profiles of all the quantities of interest follow the same trends seen for the density profiles in Fig. 12. The effect of larger losses for PINI 6 in NUBEAM is apparent in the power deposition to ions (Figs. 13(d)), whereas the power deposition to electrons and the total beam induced torque (Figs. 13(b) and (h)) remain nearly unaffected.

\section{Summary and future work}

A new beamlet-based neutral beam injection model called BBNBI was introduced. BBNBI has a more detailed geometry definition than existing neutral beam ionization codes and it can take into account ionization outside the last closed flux surface. The injector geometries of ASDEX Upgrade, DIII-D,
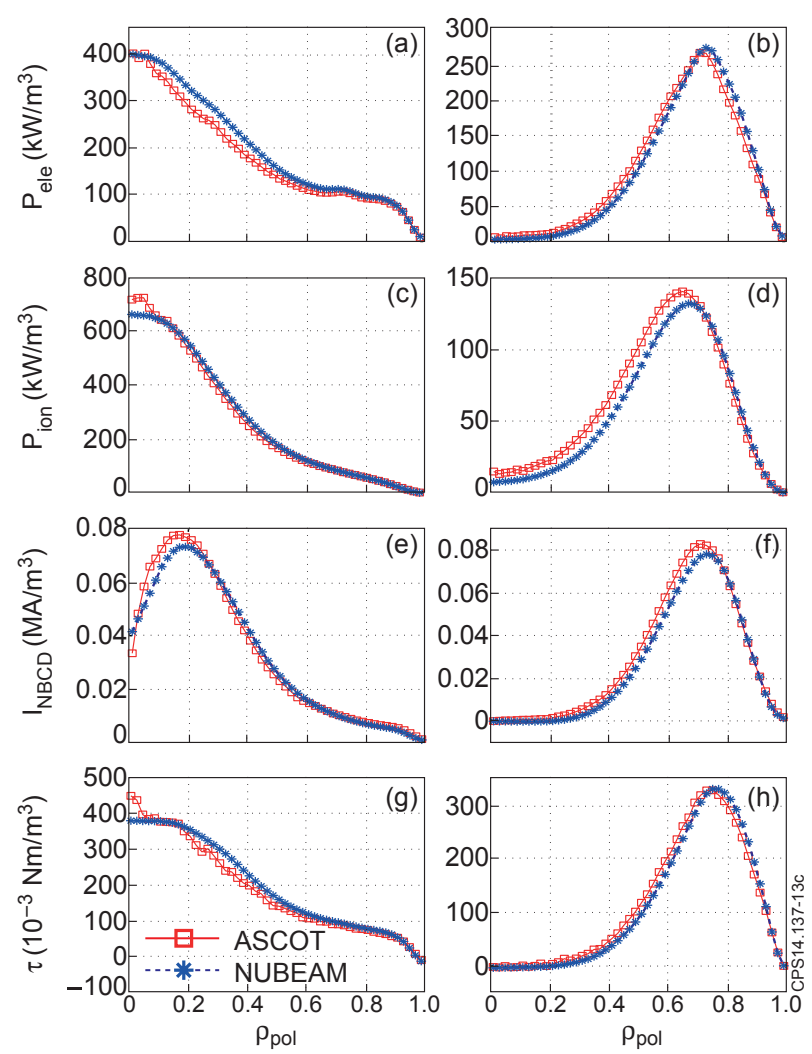

Figure 13: Power deposition from beam particles to electrons (top row) and ions (second row), the current driven by beam particles (third row) and the total torque induced by the beam particles (bottom row) in a AUG-like plasma for PINIs 4 (left column) and 6 (right column).

FAST, ITER, JET, MAST, TEXTOR, and Tore Supra have already been implemented in BBNBI, and more devices will be included as needed. BBNBI is compatible with the $\mathrm{I} / \mathrm{O}$ structures of the particle orbit following code ASCOT, which ensures consistency between the beam ionization and beam slowing down calculations. However, it can also be operated as a stand-alone tool.

Predictions of BBNBI on beam ionization were compared to those of PENCIL [9] and NUBEAM [12, 13] in axisymmetric JET- and AUG-like plasmas. First the ionization of monoenergetic $(60 \mathrm{keV} / 93 \mathrm{keV})$ neutral beams from all the eight ASDEX Upgrade PINIs was modelled with NUBEAM and BBNBI and the results of the two codes agreed very well. The radial density profile of the ionized beam particles was found to be nearly identical between the codes, and the same was true for their locations in $(R, z)$ as well as in $(x, y)$. What is more, also the distribution of ionized particles in velocity space was confirmed to agree by inspecting their pitch distribution. A similar comparison of the beam ionization was performed for the eight JET octant 8 PINIs using a more realistic beam composition with current fractions 70\%:20\%:10\% for the energy components $E_{\max }: E_{\max } / 2: E_{\max } / 3$ and $E_{\max }=100 \mathrm{keV}$. In this comparison, the distributions of ionized beam particles from BBNBI and NUBEAM were discovered to be nearly indistinguishable. The beams from PENCIL were slightly narrower, but nonetheless very similar. During the course of this work it was con- 
firmed that the initial beam ion ensemble and, hence, beam ionization has a strong impact on the beam slowing down profiles.

The first comprehensive benchmark of particle following codes ASCOT and NUBEAM and the Fokker-Planck code PENCIL was carried out. Steady-state profiles of fast ion density, power deposition, beam driven current and torque induced by the beams were compared for all JET octant 8 PINIs and all eight AUG PINIs. For JET, the profiles produced by ASCOT and NUBEAM were nearly identical for all quantities of interest. Because of the lack of ion orbit effects, PENCIL tends to give predictions that are more peaked than those of the particle following codes. For on-axis beams PENCIL is nearly in agreement with ASCOT and NUBEAM but for the off-axis beams, particularly the normal ones, the shape of the profile is different. This is caused by injected particles' wide banana orbits. In situations where the ratio of particles' orbit width and the plasma minor radius is smaller, e.g. due to higher plasma current, the discrepancies between PENCIL and particle following codes are expected to be smaller.

The benchmark between BBNBI+ASCOT and NUBEAM was repeated using an AUG-like plasma. The one-dimensional profiles of fast ion density, power deposition, beam driven current and torque predicted by the two codes were found to be in a very good agreement despite minor differences in the fast particle losses for PINIs 6 and 7. Thus, it has been shown that the results of BBNBI+ASCOT coincide with those of NUBEAM for axisymmetric plasmas for both JET and AUG. Together with earlier 3D studies [6, 7] these results further validate using BBNBI+ASCOT also for studying phenomena that require particle following in a truly three-dimensional geometry.

In the future, beam ionization on cold neutral particles could be taken into account in BBNBI. This would allow realistic simulations of the beam ionization outside and close to the last closed flux surface and the consequent fast ion losses on the limiters next to the beam port, e.g. at JET. Future work also includes using BBNBI and ASCOT to cater for the neutral beam ionization and slowing down modelling needs of European Transport Simulator (ETS) [33, 32] within the EFDA Integrated Tokamak Modelling framework (ITM) [11]. Benchmarking BBNBI and ASCOT against other similar tools adapted to the ITM standards will be an integral part of this effort.

\section{Acknowledgements}

The authors would like to thank Dr. Ian Day for invaluable discussions that helped in understanding the experimental reality of neutral beams. This work, supported by the European Communities under the contract of Association between Euratom/Tekes, was carried out within the framework of the European Fusion Development Agreement. The views and opinions expressed herein do not necessarily reflect those of the European Commission. The supercomputing resources of CSC-IT Center for Science and HPC-FF were utilized in the studies. A part of this work was carried out using the HELIOS supercomputer system at International Fusion Energy Research Centre, Aomori, Japan, under the Broader Approach collaboration between Euratom and Japan, implemented by Fusion for Energy and JAEA. The work was partially funded by the Academy of Finland projects No. 134924 and 259675.

\section{References}

[1] J. A. Heikkinen, S. K. Sipilä, T. J. H. Pättikangas, Monte Carlo simulation of runaway electrons in a toroidal geometry, Computer Physics Communications 76 (2) (1993) 215-230. doi:10.1016/0010-4655(93)90133-W. URL http://www.sciencedirect.com/science/article/ B6TJ5-46JYMP4-4T/2/fba61a679f0da244c7c01213858cd5e6

[2] J. A. Heikkinen, S. K. Sipilä, Power transfer and current generation of fast ions with large- $k_{\theta}$ waves in tokamak plasmas, Physics of Plasmas 2 (10) (1995) 3724-3733. doi:10.1063/1.871072.

URL http: //link . aip.org/link/?PHP/2/3724/1

[3] T. Kurki-Suonio, O. Asunta, T. Hellsten, V. Hynönen, T. Johnson, T. Koskela, J. Lönnroth, V. Parail, M. Roccella, G. Saibene, A. Salmi, S. Sipilä, ASCOT simulations of fast ion power loads to the plasmafacing components in ITER, Nuclear Fusion 49 (9) (2009) 095001. doi:10.1088/0029-5515/49/9/095001.

URL http://stacks.iop.org/0029-5515/49/i=9/a=095001

[4] V. Hynönen, T. Kurki-Suonio, W. Suttrop, R. Dux, K. Sugiyama, the ASDEX Upgrade Team, Surface loads and edge fast ion distribution for co- and counter-injection in ASDEX Upgrade, Plasma Physics and Controlled Fusion 49 (2) (2007) 151. URL http: //stacks . iop.org/0741-3335/49/i=2/a=006

[5] A. Salmi, T. Johnson, V. Parail, J. Heikkinen, V. Hynönen, T. P. Kiviniemi, T. Kurki-Suonio, JET EFDA Contributors, ASCOT modelling of ripple effects on toroidal torque, Contributions to Plasma Physics 48 (1-3) (2008) 77-81. doi:10.1002/ctpp.200810013.

URL http://dx.doi.org/10.1002/ctpp. 200810013

[6] A. T. Salmi, T. Tala, G. Corrigan, C. Giroud, J. Ferreira, J. Lönnroth, P. Mantica, V. Parail, M. Tsalas, T. W. Versloot, P. C. de Vries, K.-D. Zastrow, EFDA JET Contributors, NBI torque in the presence of magnetic field ripple: experiments and modelling for JET, Plasma Physics and Controlled Fusion 53 (8) (2011) 085005.

URL http: //stacks.iop.org/0741-3335/53/i=8/a=085005

[7] G. Kramer, A. McLean, N. Brooks, R. Budny, X. Chen, W. Heidbrink, T. Kurki-Suonio, R. Nazikian, T. Koskela, M. Schaffer, K. Shinohara, J. Snipes, M. V. Zeeland, Simulation of localized fast-ion heat loads in test blanket module simulation experiments on diii-d, Nuclear Fusion 53 (12) (2013) 123018.

URL http: //stacks . iop.org/0029-5515/53/i=12/a=123018

[8] D. Moseev, F. Meo, S. B. Korsholm, T. Koskela, M. Albergante, O. Asunta, H. Bindslev, A. Bürger, V. Furtula, M. Y. Kantor, F. Leipold, P. K. Michelsen, S. K. Nielsen, M. Salewski, O. Schmitz, M. Stejner, E. Westerhof, the TEXTOR team, Comparison of measured and simulated fast ion velocity distributions in the TEXTOR tokamak, Plasma Physics and Controlled Fusion 53 (10) (2011) 105004.

URL http: //stacks.iop.org/0741-3335/53/i=10/a=105004

[9] C. Challis, J. Cordey, H. Hamnén, P. Stubberfield, J. Christiansen, E. Lazzard, D. Muir, D. Stork, E. Thompson, Non-inductively driven currents in jet, Nuclear Fusion 29 (1989) 563-570.

[10] G. G. Lister, FAFNER: A Fully 3-D Neutral Beam Injection Code Using Monte Carlo Methods, Technical Report IPP 4/222, Max-Planck-Institut für Plasmaphysik (1985).

[11] B. Guillerminet, M. Airaj, P. Huynh, G. Huysmans, F. Iannone, F. Imbeaux, J. Lister, G. Manduchi, P. Strand, Integrated tokamak modelling: Infrastructure and software integration project, Fusion Engineering and Design 83 (2008) 442-447.

[12] R. Goldston, D. McCune, H. Towner, S. Davis, R. Hawryluk, G. Schmidt, New techniques for calculating heat and particle source rates due to neutral beam injection in axisymmetric tokamaks, Journal of Computational Physics 43 (1) (1981) 61 - 78. doi:10.1016/0021-9991(81)90111-X. URL http://www.sciencedirect.com/science/article/ pii/002199918190111X

[13] A. Pankin, D. McCune, R. Andre, G. Bateman, A. Kritz, The tokamak Monte Carlo fast ion module NUBEAM in the national transport code collaboration library, Computer Physics Communications 159 (3) (2004) 157 - 184. doi:10.1016/j.cpc.2003.11.002.

URL http://www.sciencedirect.com/science/article/ pii/S0010465504001109 
[14] R. Hawryluk, An empirical approach to tokamak transport, in: B. Coppi, G. Leotta, D. Pfirsch, R. Pozzoli, E. Sindoni (Eds.), Physics of Plasmas Close to Thermonuclear Conditions, Volume 1, Vol. 1, Pergamon Press, Oxford, UK, 1981, pp. 19-46. URL http://w3.pppl.gov/ pshare/transp/papers/Hawryluk.pdf

[15] M. Kraus, Heiz- und stromprofile bei neutralteilcheninjektion in tokamakplasmen, Technical Report IPP 5/122, Max-Planck-Institut für Plasmaphysik, http: //edoc. $\mathrm{mpg}$. de/get . epl?fid=71462\&did=478136\&ver $=0$ (2010).

[16] T. Inoue, E. D. Pietro, M. Hanada, R. Hemsworth, A. Krylov, V. Kulygin, P. Massmann, P. Mondino, Y. Okumura, A. Panasenkov, E. Speth, K. Watanabe, Design of neutral beam system for iter-feat, Fusion Engineering and Design 56-57 (0) (2001) 517 - 521. doi:10.1016/S09203796(01)00339-8

URL http://www.sciencedirect.com/science/article/ pii/S0920379601003398

[17] Design Description Document (WBS 5.3) Neutral Beam Heating \& Current Drive (NBH\&CD) SYSTEM, Appendix 2, Beam Alignment and Transmission, ITER, 2002.

[18] S. Suzuki, T. Shirai, M. Nemoto, K. Tobita, H. Kubo, T. Sugie, A. Sakasai, Y. Kusama, Attenuation of high-energy neutral hydrogen beams in highdensity plasmas, Plasma Physics and Controlled Fusion 40 (12) (1998) 2097-2111. doi:10.1088/0741-3335/40/12/009.

URL http: //stacks.iop.org/0741-3335/40/2097

[19] J. Ongena, M. Evrard, D. McCune, Numerical transport codes, Fusion Science and Technology 63 (2T) (2008) 367-376.

[20] G. Tardini, J. Hobirk, V. Igochine, C. Maggi, P. Martin, D. McCune, A. Peeters, A. Sips, A. Stäbler, J. Stober, the ASDEX Upgrade Team, Thermal ions dilution and ITG suppression in ASDEX Upgrade ion ITBs, Nuclear Fusion 47 (4) (2007) 280. URL http: //stacks . iop.org/0029-5515/47/i=4/a=006

[21] G. Tardini, C. Höhbauer, R. Fischer, R. Neu, the ASDEX Upgrade Team, Simulation of the neutron rate in ASDEX Upgrade H-mode discharges, Nuclear Fusion 53 (6) (2013) 063027.

URL http: //stacks. iop.org/0029-5515/53/i=6/a=063027

[22] T. Tala, K.-D. Zastrow, J. Ferreira, P. Mantica, V. Naulin, A. G. Peeters, G. Tardini, M. Brix, G. Corrigan, C. Giroud, D. Strintzi, Evidence of inward toroidal momentum convection in the JET tokamak, Phys. Rev. Lett. 102 (2009) 075001. doi:10.1103/PhysRevLett.102.075001. URL http: //link.aps.org/doi/10.1103/PhysRevLett.102.075001

[23] C. Hellesen, M. Albergante, E. A. Sundén, L. Ballabio, S. Conroy, G. Ericsson, M. G. Johnson, L. Giacomelli, G. Gorini, A. Hjalmarsson, I. Jenkins, J. Källne, E. Ronchi, H. Sjöstrand, M. Tardocchi, I. Voitsekhovitch, M. Weiszflog, JET-EFDA contributors, Neutron spectroscopy measurements and modeling of neutral beam heating fast ion dynamics, Plasma Physics and Controlled Fusion 52 (8) (2010) 085013 URL http: //stacks . iop.org/0741-3335/52/i=8/a=085013

[24] G. Tardini, J. Ferreira, P. Mantica, A. Peeters, T. Tala, K. Zastrow, M. Brix, C. Giroud, G. Pereverzev, JET-EFDA contributors, Angular momentum studies with NBI modulation in jet, Nuclear Fusion 49 (8) (2009) 085010 . URL http: //stacks . iop.org/0029-5515/49/i=8/a=085010

[25] ADAS, Atomic data and analysis software, http://www.adas.ac.uk/.

[26] R. Janev, C. Boley, D. Post, Penetration of energetic neutral beams into fusion plasmas, Nuclear Fusion 29 (12) (1989) 2125 URL http: //stacks . iop.org/0029-5515/29/i=12/a=006

[27] A. H. Boozer, G. Kuo-Petravic, Monte carlo evaluation of transport coefficients, Physics of Fluids 24 (5) (1981) 851-859. doi:10.1063/1.863445. URL http: //link. aip.org/link/?PFL/24/851/1

[28] A. Mück, T. P. Goodman, M. Maraschek, G. Pereverzev, F. Ryter, H. Zohm, ASDEX Upgrade Team, Sawtooth control experiments on asdex upgrade, Plasma Physics and Controlled Fusion 47 (10) (2005) 1633. URL http://stacks.iop.org/0741-3335/47/i=10/a=004

[29] M.-L. Mayoral, V. Bobkov, A. Czarnecka, I. Day, A. Ekedahl, P. Jacquet, M. Goniche, R. King, K. Kirov, E. Lerche, J. Mailloux, D. V. Eester, O. Asunta, C. Challis, D. Ciric, J. Coenen, L. Colas, C. Giroud, M. Graham, I. Jenkins, E. Joffrin, T. Jones, D. King, V. Kiptily, C. Klepper, C. Maggi, R. Maggiora, F. Marcotte, G. Matthews, D. Milanesio, I. Monakhov, M. Nightingale, R. Neu, J. Ongena, T. Ptterich, V. Riccardo, F. Rimini, J. Strachan, E. Surrey, V. Thompson, G. V. Rooij, J. E. Contributors,
On the challenge of plasma heating with the jet metallic wall, Nuclear Fusion 54 (3) (2014) 033002

URL http: //stacks . iop.org/0029-5515/54/i=3/a=033002

[30] S. Wiesen, et al., JINTRAC-JET modelling suite, JET ITC-Report (2008) http://www.eirene.de/JINTRAC_Report_2008.pdf.

[31] S. Wiesen, S. Brezinsek, A. Järvinen, T. Eich, W. Fundamenski, A. Huber, V. Parail, G. Corrigan, N. Hayashi, J. contributors, Integrated modelling of a JET type-I ELMy H-mode pulse and predictions for ITER-like wall scenarios, Plasma Physics and Controlled Fusion 53 (12) (2011) 124039. URL http: //stacks . iop.org/0741-3335/53/i=12/a=124039

[32] D. Coster, V. Basiuk, G. Pereverzev, D. Kalupin, R. Zagórski, R. Stankiewicz, P. Huynh, F. Imbeaux, The european transport solver, IEEE Transactions on Plasma Science 38 (9) (2010) 2085-2092. doi:10.1109/TPS.2010.2056707.

[33] D. Kalupin, I. Ivanova-Stanik, I. Voitsekhovitch, J. Ferreira, D. Coster, L. Alves, T. Aniel, J. Artaud, V. Basiuk, J. P. Bizarro, R. Coelho, A. Czarnecka, P. Huynh, A. Figueiredo, J. Garcia, L. Garzotti, F. Imbeaux, F. Köchl, M. Nave, G. Pereverzev, O. Sauter, B. Scott, R. Stankiewicz, P. Strand, I.-T. contributors, JET-EFDA Contributors, Numerical analysis of JET discharges with the european transport simulator, Nuclear Fusion 53 (12) (2013) 123007.

URL http://stacks.iop.org/0029-5515/53/i=12/a=123007

[34] D. Mikkelsen, C. Singer, Optimization of steady-state beam-driven tokamak reactors, Princeton Plasma Physics Laboratory, Princeton, New Jersey, 1982. doi:10.2172/6772488.

URL http: //www.osti.gov/scitech/servlets/purl/6772488

[35] Y. Lin-Liu, F. Hinton, Trapped electron correction to beam driven current in general tokamak equilibria, Physics of Plasmas 4 (1997) 4179.

[36] J. Cordey, F. Haas, Aspects of the equilibrium and stability of counterstreaming-ion tokamaks, Nuclear Fusion 16 (4) (1976) 605. URL http: //stacks . iop.org/0029-5515/16/i=4/a=006

[37] R. M. McDermott, C. Angioni, R. Dux, E. Fable, T. Pütterich, F. Ryter, A. Salmi, T. Tala, G. Tardini, E. Viezzer, the ASDEX Upgrade Team, Core momentum and particle transport studies in the ASDEX Upgrade tokamak, Plasma Physics and Controlled Fusion 53 (12) (2011) 124013. URL http://stacks.iop.org/0741-3335/53/i=12/a=124013

[38] K.-D. Zastrow, W. Core, L.-G. Eriksson, M. V. Hellermann, A. Howman, R. König, Transfer rates of toroidal angular momentum during neutral beam injection, Nuclear Fusion 38 (2) (1998) 257.

URL http://stacks. iop.org/0029-5515/38/i=2/a=309 